\title{
NF-кB1 promoter-94ins/delATTG polymorphisms correlate with the acute exacerbation of chronic obstructive pulmonary disease
}

\author{
Xun Meng ${ }^{1 *}$, Shao-Qiang Zheng ${ }^{2 *}$, Jin-Hong Wang ${ }^{2}$, Tao Zhang ${ }^{1}$ \\ ${ }^{1}$ Department of ENT, The First Affiliated Hospital, Jinan University, Guangzhou 510630, China; ${ }^{2}$ Department of Respiratory Medicine, The Third \\ Affiliated Hospital of Southern Medical University Guangzhou, Guangzhou 510630, China \\ Contributions: (I) Conception and design: JH Wang; (II) Administrative support: T Zhang; (III) Provision of study materials or patients: JH Wang, \\ SQ Zheng; (IV) Collection and assembly of data: X Meng, SQ Zheng; (V) Data analysis and interpretation: JH Wang, X Meng, SQ Zheng; (VI) \\ Manuscript writing: All authors; (VII) Final approval of manuscript: All authors. \\ \#These authors contributed equally to this work. \\ Correspondence to: Tao Zhang. Department of ENT, The First Affiliated Hospital of Jinan University, Guangzhou 510630, China. \\ Email: tzhangt@jnu.edu.cn; Jin-Hong Wang. Department of Respiratory Medicine, The Third Affiliated Hospital of Southern Medical University \\ Guangzhou, Guangzhou 510630, China. Email: leechy911@126.com.
}

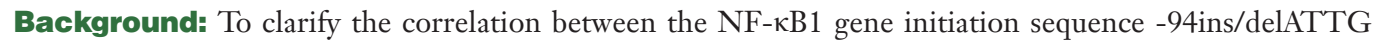
polymorphisms and the acute exacerbation of chronic obstructive pulmonary disease (AECOPD).

Methods: Blood samples of 260 AECOPD patients were collected from September 2013 to September 2015 in the department of respiratory medicine, the Third Affiliated Hospital of Southern Medical University. Blood samples of 260 healthy subjects were collected as a control group. DNA was extracted using genomic DNA extraction kits and analyzed on a DNA quantitative analyzer. Data analysis was performed using Rotor-Gene (60001.7) to determine genotypes. SPSS20.0 was used to compare -94ins/ delATTG polymorphisms between patients and healthy subjects. The relationship between the promoter sequence -94ins/delATTG of NF-кB1 genotypes and AECOPD were further analyzed.

Results: We detected ins/ins, insertion or deletion (ins/del) and del/del genotypes from both the AECOPD and healthy control groups. The distribution of the three genotypes were consistent with the Hardy-Weinberg equilibrium law. The composition ratios of ins/ins, ins/del, del/del genotype distributions differed between AECOPD and control groups $(\mathrm{P}<0.05)$. The differences in ins/ins, ins/del and del/del genotype distributions between the two groups also significantly differed $(\mathrm{P}<0.05)$. The distribution of allele frequencies was comparable between the groups $(\mathrm{P}>0.05)$. The distribution ratio showed no relevance to the smoking index and clinical phenotypes of AECOPD patients, whether carrying ins/ins + ins/del genotypes or del/del genes $(\mathrm{P}>0.05)$. Compared to AECOPD patients with del/del genotypes, AECOPD patients with ins/ins + ins/del genotypes had a lower body mass index (BMI), a higher COPD assessment test (CAT) score, a larger number of acute episodes and longer hospital stays $(\mathrm{P}<0.05)$.

Conclusions: The detection of the -94ins/delATTG polymorphism in patients with AECOPD can predict disease prognosis. The BMI of patients with AECOPD was significantly lower in patients carrying the -94insATTG gene. Gene detection is therefore important in patients carrying ins/ins or ins/del genotypes following admission.

Keywords: NF-кB1; -94ins/delATTG; acute exacerbation of chronic obstructive pulmonary disease (AECOPD); gene polymorphism analysis

Submitted Sep 12, 2019. Accepted for publication Nov 12, 2019.

doi: $10.21037 /$ jtd.2019.11.37

View this article at: http://dx.doi.org/10.21037/jtd.2019.11.37

(C) Journal of Thoracic Disease. All rights reserved. 


\section{Introduction}

Chronic obstructive pulmonary disease (COPD) is a major threat to human health. Epidemiological data (1) shows that the prevalence of COPD patients aged above 40 years old in China is $8.2 \%$ and each year COPD patients encounter acute exacerbation (AE) on 0.5 to 3.5 occasions. Studies (2) have confirmed that acute exacerbation of chronic obstructive pulmonary disease (AECOPD) is an important cause of hospitalization and death in patients with COPD. This disease represents a serious economic and social burden.

The pathogenesis of COPD remains unclear but is known to involve environmental and genetic factors (3) and inflammatory cells, inflammatory mediators and cytokines. As a pivotal signaling pathway, NF- $\mathrm{\kappa B}$ plays an important role in COPD occurrence. Pierrou et al. (4) revealed that the bronchial epithelial cells of COPD patients overexpress a large number of transcription factors including $\mathrm{NF}-\kappa \mathrm{B}$. Brown et al. (5) found that the excessive expression or activation of NF- $\mathrm{KB} 1$ in patients with COPD and lung cancer led to exacerbated inflammatory responses, making COPD patients more prone to lung cancer.

It is known now that an ATTG insertion or deletion (ins/ del) at the $94 \mathrm{bp}$ region upstream of the NF-kB p50 (NF$\kappa \mathrm{B} 1$ gene) transcription initiation site influences COPD development. Studies (6) have shown that the NF-кB1 gene initiation sequence -94ins/delATTG polymorphism influences the expression of NF- $\kappa B$ which in turn affects disease progression. Others (7) have confirmed that the NFкB1 gene initiation sequence -94ins/delATTG represents a susceptibility gene for chronic lung disease, but the correlation between NF- $\mathrm{KB} 1$ gene polymorphisms and the $\mathrm{AE}$ of chronic lung disease has not been defined.

In this study, we used polymerase chain reaction high resolution melt (PCR-HRM) to assess the distribution of NF- $\mathrm{BB} 1$ gene initiation sequence -94ins/delATTG polymorphisms amongst the Chinese Han population with COPD. We further assessed the relationship between polymorphisms and the AECOPD.

\section{Methods}

\section{Subjects}

A total of 260 patients with COPD were recruited from the outpatient and inpatient clinics of the Department of
Respiratory Medicine of the Third Affiliated Hospital of the Southern Medical University during September 2013 to September 2015. Patients were diagnosed as COPD by following the global initiative for chronic obstructive lung disease (GOLD) diagnostic criteria (3).

The diagnostic criteria were as follows: AE diagnosis criteria: COPD patients diagnosed when their respiratory symptoms continued to deteriorate abnormally with a requirement for other medication regimens (e.g., fluticasone/salmeterol in stable COPD; intravenous hexadecadrol for AECOPD). During disease progression, patients often present with short-term coughs, expectoration, a shortness of breath, wheezing phlegm, increased pus and phlegm or mucus purulent sputum accompanied by fever and other aggravations. A total of 260 healthy subjects were selected as the control group. Body mass index (BMI) and COPD assessment test (CAT) scores were assessed in all patients.

\section{Inclusion criteria}

Met the above diagnostic criteria; unrelated; provided informed consent and cooperated with all experiments.

\section{Exclusion criteria}

Patients with respiratory system diseases such as bronchiectasis, pulmonary fibrosis, sarcoidosis, bronchial asthma and inherited diseases such as diabetes, heart disease and rheumatic arthritis.

All selected patients and healthy controls were authorized by the Ethics Committee of clinical trials of the Third Affiliated Hospital of Southern Medical University (2016-clinic-042).

\section{Reagents and instruments}

DNA extraction kits were purchased from Fermentas Co., (K0512, Lithuania); Type-it HRM PCR kits were purchased from Qiagen Co., (206542, Germany). Rotor-Gene Q Analyzer kits were purchased from Qiagen Co., (RotorGene Q, Germany). DNA quantitative analyzer kits were purchased from Fermentas Co., Lithuania.

\section{Materials and methods}

Genomic DNA extraction and quantification

A total of $1 \mathrm{~mL}$ of EDTA anticoagulant venous blood was 
Table 1 Characteristics of the AECOPD and control group

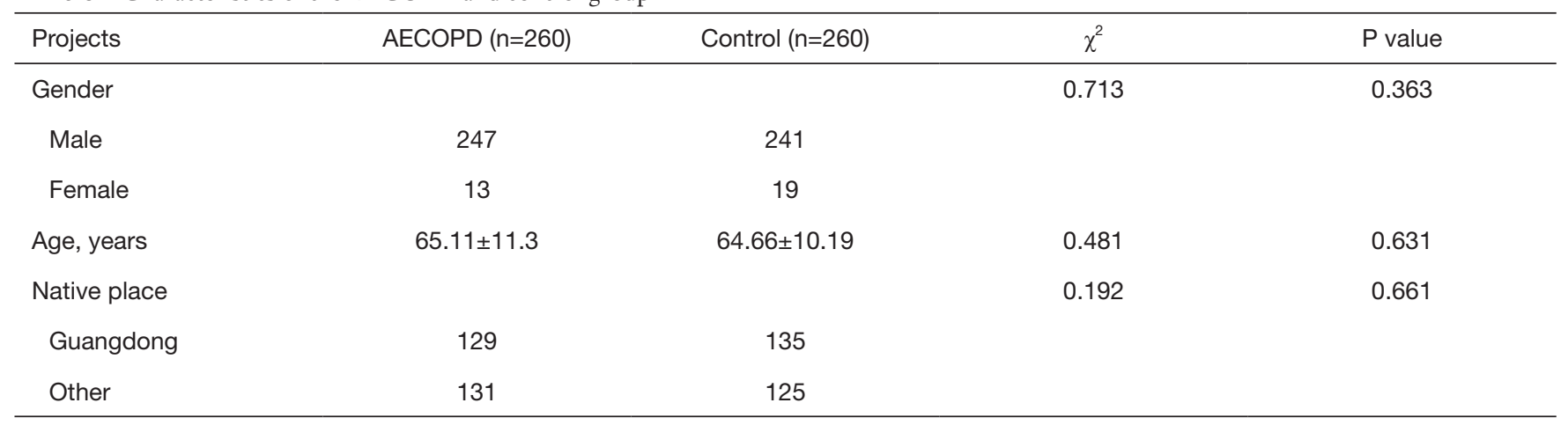

AECOPD, acute exacerbation of chronic obstructive pulmonary disease.

collected from both patient and control groups. DNA was extracted using genomic DNA extraction kits according to the manufacturer's instruction. The concentration and purity of the extracted genomic DNA were analyzed on a DNA quantitative analyzer. DNA samples were diluted to $30 \mathrm{ng} / \mu \mathrm{L}$ and stored at $-20^{\circ} \mathrm{C}$.

PCR-HRM was used to detect NF-кB1 gene initiation sequences and regional polymorphisms. PCR sequences were as previously described (8). Upstream primer: 5'-CATGACTCTATCAGCGGCACT-3', downstream primer: 5'-GGCTCTGGCTTCCTAGCAG-3'; product length: 152 bps. Primers were synthesized by Weijiki (Shanghai) Trading Co., Ltd. PCRs were performed using Type-it HRM PCR kits. PCR reaction conditions were as follows: initial denaturation for $5 \mathrm{~min}$ at $96^{\circ} \mathrm{C}$; denaturation for $20 \mathrm{~s}$ at $96^{\circ} \mathrm{C} ; 20 \mathrm{~s}$ annealing at $57^{\circ} \mathrm{C}$; extension for $20 \mathrm{~s}$ at $72{ }^{\circ} \mathrm{C}$; 40 cycles. HRM analysis and PCR reactions were performed simultaneously. HRM analysis conditions: $95^{\circ} \mathrm{C}$ for $5 \mathrm{~min} ; 40{ }^{\circ} \mathrm{C}$ for $2 \mathrm{~min} ; 6{ }^{\circ} \mathrm{C}$ for $1 \mathrm{~min}$, and collect data of melting curves at $0.1{ }^{\circ} \mathrm{C} / \mathrm{s}$ from $72{ }^{\circ} \mathrm{C}$ to $95{ }^{\circ} \mathrm{C}$. Each HRM analysis assessed the ins/ins and del/del genotypes simultaneously. Rotor-Gene 60001.7 was used to analyze the real-time data and determine the genotype. A total of $5 \%$ of the samples were randomly selected for direct sequencing to verify the HRM technology.

\section{Statistical analysis}

Data were processed using SPSS 20.0. The $\chi^{2}$ test was for group comparisons of the counting data. When the theoretical frequency was $<5$, a Fisher's exact test was used for comparisons. Numerical data are presented as mean \pm standard deviation (SD) $(\overline{\mathrm{x}} \pm \mathrm{s})$. Non-normal data were indicated by the median and quartile spacing. A one- way analysis of variance (ANOVA) was used to compare measurement data between the groups. A significance level of $\alpha$ is 0.05 was employed.

\section{Results}

\section{General information}

No significant differences in age, sex and location were observed between the AECOPD and control groups $(\mathrm{P}>0.05$, Table 1). The median smoking index (per cigarette/1 year) amongst the control group was $301.53 \pm 287.59$ (pack/years).

\section{NF-кB1 gene initiation sequence regional polymorphism analysis}

Samples from experimental and control groups were compared for NF- $\kappa \mathrm{B} 1$ activation by PCR-HRM. The results showed that both the AECOPD and healthy control group showed detectable ins/ins, ins/del and del/del genotypes (Figures 1,2). Genetic balance analysis showed that the distribution of the three genotypes were consistent with the Hardy-Weinberg equilibrium law in both groups. The number of patients who carried ins/ins, ins/del and del/ del genotypes were 93, 138 and 29 in the AECOPD group and 102, 106 and 52 in the control group respectively. The different distributions of the two groups were statistically significant $(\mathrm{P}<0.05$, Table 2). Further analysis showed that the distribution of the composition ratio of ins/ins + ins/ del and del/del between groups were statistically significant $(\mathrm{P}<0.05$, Table 2). However, the distribution of the composition ratio of ins and del alleles did not significantly differ between groups ( $\mathrm{P}>0.05$, Table 2$)$. 


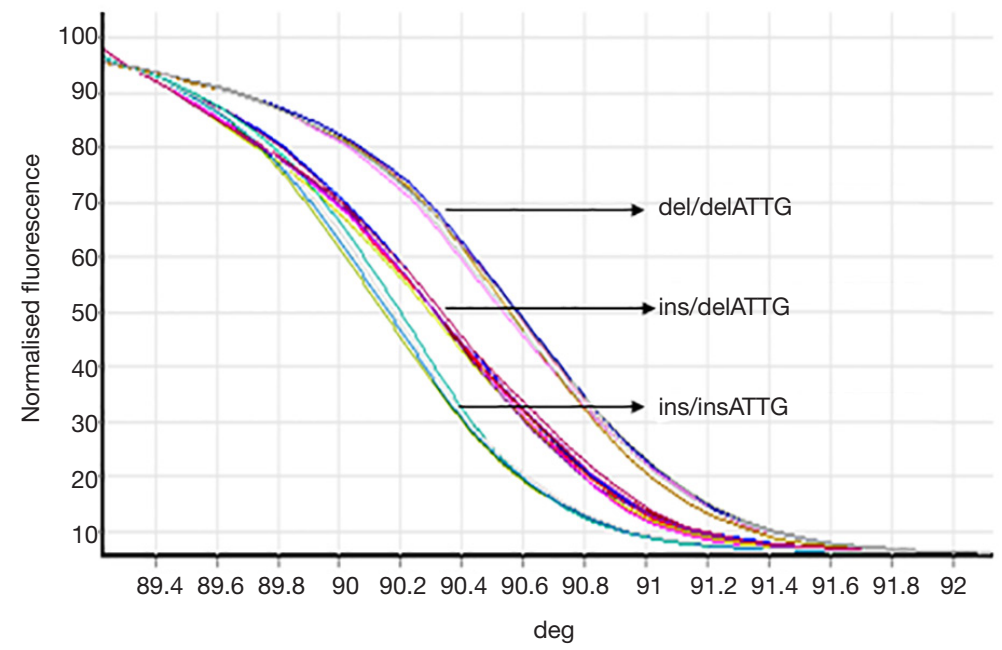

Figure 1 HRM standard view. HRM, high resolution melt. deg, degree.

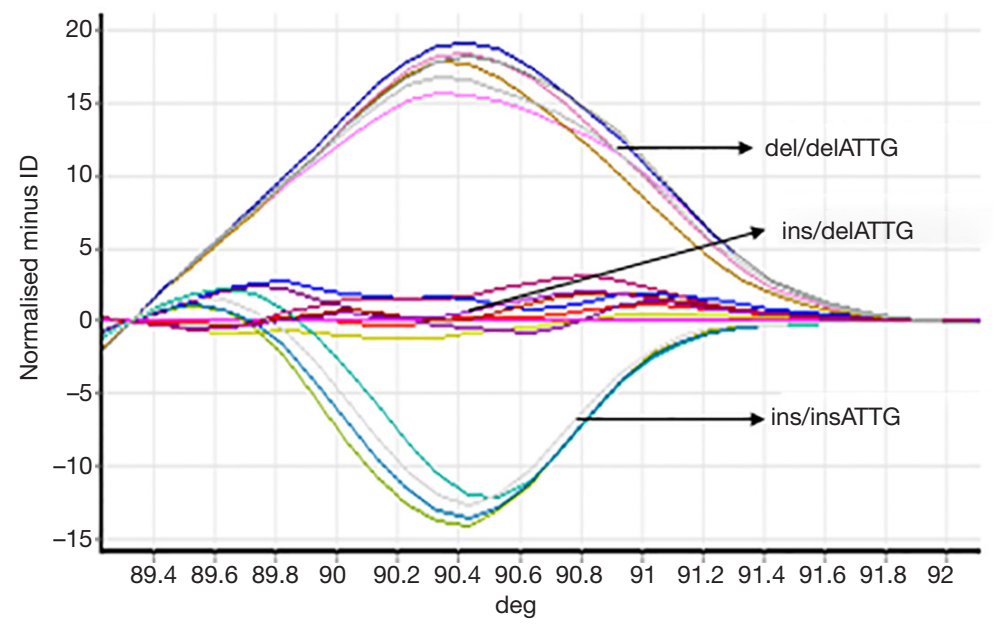

Figure 2 Differences in view of 3 genotypes in HRM (set ins/delATTG genotype as standard). HRM, high resolution melt; deg, degree.

\section{Correlation analysis of NF- $\mathrm{NB1}$ gene initiation -94ins/ delATTG and AECOPD}

\section{General information of the AECOPD patients}

General information analysis showed no significant differences between ins/ins + ins/del and del/del in terms of patient gender, age and native location $(\mathrm{P}>0.05$, Table 3$)$.

Analysis of the clinical features of the ins/ins + ins/del and del/del genes in AECOPD patients

Whether the AECOPD patients carried ins/ins + ins/ del or del/del genes were next assessed. The distribution composition was independent of the smoking index and clinical phenotype $(\mathrm{P}>0.05)$ as the control group had the same smoking history. AECOPD patients who carried the ins/ins + ins/del gene had a lower BMI, higher CAT scores, a higher number of acute episodes and longer hospital stays than the AECOPD patients with the del/del gene $(\mathrm{P}<0.05$, Table 4).

\section{Discussion}

A large number of studies (5,9-10) have shown that NF- $\kappa \mathrm{B}$ is associated with COPD associated airway inflammation, which regulates the expression of pro-inflammatory genes that regulate cell proliferation, apoptosis and immune 
Table 2 Distribution of NF-кB1 gene initiation sequences -94ins/delATTG and alleles in AECOPD and control groups

\begin{tabular}{|c|c|c|c|c|}
\hline Genotype/allelic & AECOPD & Control & $\chi^{2}$ & $P$ value \\
\hline del/del & 29 & 52 & 11.143 & 0.004 \\
\hline ins/del & 138 & 106 & & \\
\hline ins/ins & 93 & 102 & & \\
\hline $\mathrm{del} / \mathrm{del}$ & 29 & 52 & & \\
\hline Allelic & & & 0.792 & 0.374 \\
\hline del & 196 & 210 & & \\
\hline ins & 324 & 310 & & \\
\hline
\end{tabular}

AECOPD, acute exacerbation of chronic obstructive pulmonary disease.

Table 3 Correlation analysis between AECOPD patients' characteristics and -94ins/delATTG genotype

\begin{tabular}{|c|c|c|c|c|}
\hline Projects & \multicolumn{2}{|c|}{ Genotype } & $\chi^{2}$ & $P$ value \\
\hline Gender & & & 1.963 & 0.161 \\
\hline Male & 26 & 221 & & \\
\hline Female & 3 & 10 & & \\
\hline Native place & & & 0.886 & 0.347 \\
\hline Guangdong & 12 & 117 & & \\
\hline Other & 17 & 114 & & \\
\hline
\end{tabular}

AECOPD, acute exacerbation of chronic obstructive pulmonary disease.

Table 4 Analysis of the clinical characteristics between the NF-кB1 the gene initiation sequence -94ins/delATTG genotype and AECOPD

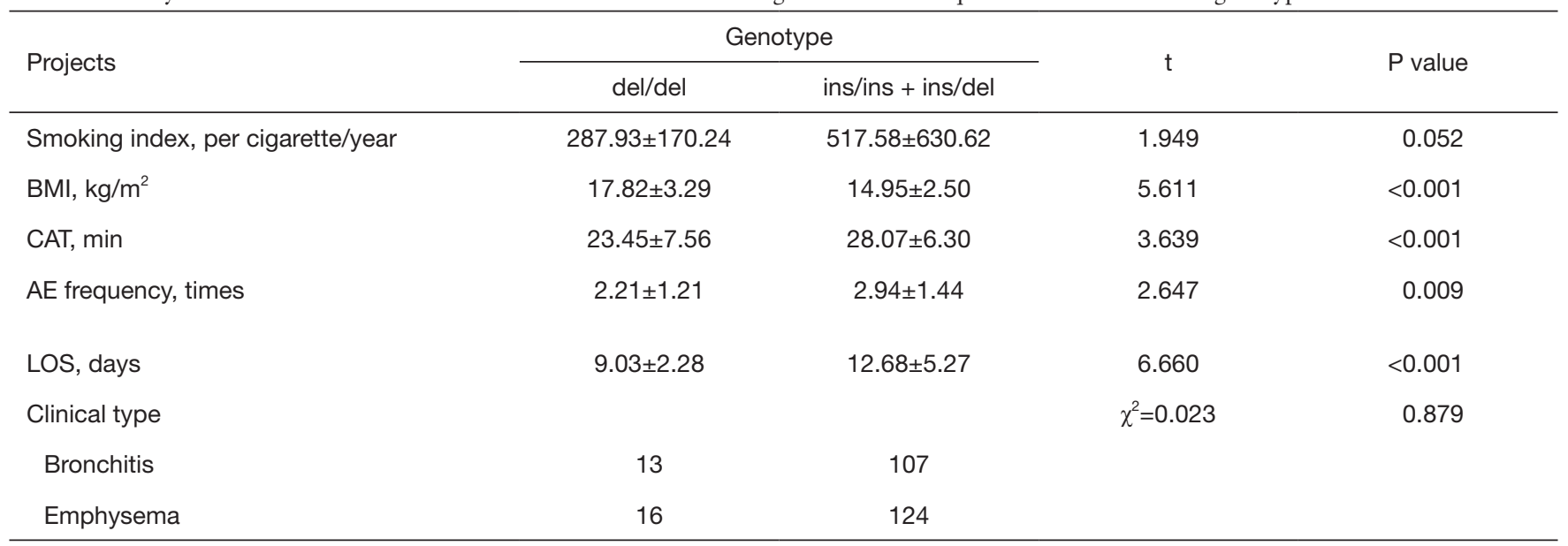

AECOPD, acute exacerbation of chronic obstructive pulmonary disease; BMI, body mass index; CAT, COPD assessment test; AE, acute exacerbation; LOS, length of stay. 
responses. Activated NF- $\mathrm{KB}$ delays neutrophil apoptosis and enhances the inflammatory response. In this study, ins/ins, ins/del and del/del were detected from AECOPD patients the majority of which carried ins/ins and ins/del. These results were consistent with studies by Huang and colleagues (11) who found that the lung function of the Chinese Han population with -94ins/insATTG genes or ins/delATTG genes showed reduced lung function (FEV1) that those carrying del/delATTG genes. The results of this study indicated that the COPD patients with -94ins/ insATTG or -94ins/delATTG gene sequences had more severe disease with longer hospital stays. It is speculated that carrying the -94insattg gene increases the expression of NF- $\mathrm{\kappa B}$ and influences the intensity of the inflammatory response. Karban et al. (6) confirmed that the NF-кB1 gene initiation sequence -94insATTG could increase the transcription capacity of NF- $\kappa B 1$ by $\sim 2$-fold, whilst the delATTG sequence reduced the transcription intensity of $\mathrm{NF}-\kappa \mathrm{B} 1$ in vitro. Adamzik et al. (12) found that the gene initiation sequence of NF- $\mathrm{KB} 1$ (-94ins/delATTG) was closely related to the severity of acute respiratory distress syndrome (ARDS), and patients with ARDS with -94del/ delATTG genes had a lower incidence of lung injury, indicating poor prognosis.

We observed no differences in terms of smoking history (pack years) between those carrying del/del genotypes and those carrying ins/ins +ins/del genotypes. However, Szulakowski et al. (13) showed that the transcription of $\mathrm{NF}-\kappa \mathrm{B}$ in lung tissue and phlegm of COPD patients was higher than non-smokers. The various oxides in cigarettes activate NF- $\mathrm{KB}$ and $\mathrm{AP}-1$ signaling to induce the expression of various cytokines and participate in the inflammatory processes of lung tissue. The results of the study were inconsistent with previous proteomic analysis, and thus require further verification (14).

We found that the BMI was an important indicator of the long-term prognosis of patients with COPD and thus an indicator of long-term survival. Patients with a low BMI tended to display more severe symptoms and higher mortality (15). We found that the BMI of patients with AECOPD was significantly lower than patients with the -94insATTG gene. We hypothesized that a correlation between body weight and the -94insATTG gene exists, which requires further confirmation.

\section{Conclusions}

We found that the NF- $\mathrm{BB} 1$ initiation sequence -94ins/
delATTG is a susceptible gene for the development of AECOPD. As a result, monitoring the polymorphism permits the ability to prevent the AECOPD, providing a theoretical basis for individualized treatments. However, the sample size of this study was small and studies with multicenter large sample sizes in combination with proteomic analysis are required to confirm the role of the NF- $\mathrm{kB} 1$ gene initiation sequence -94ins/delATTG during the AECOPD.

\section{Acknowledgments}

None.

\section{Footnote}

Conflicts of Interest: The authors declare that they have no conflicts of interest.

Ethical Statement: The authors are accountable for all aspects of the work in ensuring that questions related to the accuracy or integrity of any part of the work are appropriately investigated and resolved. All selected patients and healthy controls were authorized by the ethics committee of clinical trials of the third affiliated hospital of Southern Medical University (2016-clinic-042).

\section{References}

1. Zhong N, Wang C, Yao W, et al. Prevalence of chronic obstructive pulmonary disease in China: a large, population-based survey. Am J Respir Crit Care Med 2007;176:753-60.

2. Perera PN, Armstrong EP, Sherrill DL, et al. Acute exacerbations of COPD in the United States: inpatient burden and predictors of costs and mortality. COPD 2012;9:131-41.

3. Vestbo J, Hurd SS, Agustí AG, et al. Global strategy for the diagnosis, management, and prevention of chronic obstructive pulmonary disease. Am J Respir Crit Care Med 2013. doi: 10.1164/rccm.201204-0596PP.

4. Pierrou S, Broberg P, O'Donnell RA, et al. Expression of genes involved in oxidative stress responses in airway epithelial cells of smokers with chronic obstructive pulmonary disease. Am J Respir Crit Care Med 2007;175:577-86.

5. Brown V, Elborn JS, Bradley J, et al. Dysregulated apoptosis and NFkappaB expression in COPD subjects. 
Respir Res 2009;10:24.

6. Karban AS, Okazaki T, Panhuysen CI, et al. Functional annotation of a novel NFKB1 promoter polymorphism that increases risk for ulcerative colitis. Hum Mol Genet 2004;13:35-45.

7. Korytina GF, Akhmadishina LZ, Kochetova OV, et al. Inflammatory and immune response genes polymorphisms are associated with susceptibility to chronic obstructive pulmonary disease in Tatars population from Russia. Biochem Genet 2016;54:388-412.

8. Santos DG, Resende MF, Mill JG, et al. Nuclear factor (NF) kappaB polymorphism is associated with heart function in patients with heart failure. BMC Med Genet 2010;11:89.

9. Imanifooladi AA, Yazdani S, Nourani MR. The role of nuclear factor-kappaB in inflammatory lung disease. Inflamm Allergy Drug Targets 2010;9:197-205.

10. Liu FT, Jia L, Wang P, et al. STAT3 and NF-кB cooperatively control in vitro spontaneous apoptosis and poor chemo-responsiveness in patients with chronic

Cite this article as: Meng X, Zheng SQ, Wang JH, Zhang T.

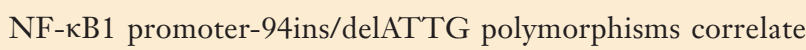
with the acute exacerbation of chronic obstructive pulmonary disease. J Thorac Dis 2019;11(12):5433-5439. doi: 10.21037/ jtd.2019.11.37 lymphocytic leukemia. Oncotarget 2016;7:32031-45.

11. Huang D, Yang L, Liu Y, et al. Functional polymorphisms in $\mathrm{NF \kappa B} 1 / \mathrm{I} \kappa \mathrm{B} \alpha$ predict risks of chronic obstructive pulmonary disease and lung cancer in Chinese. Hum Genet 2013;132:451-60.

12. Adamzik M, Frey UH, Rieman K, et al. Insertion/deletion polymorphism in the promoter of NFKB1 influences severity but not mortality of acute respiratory distress syndrome. Intensive Care Med 2007;33:1199-203.

13. Szulakowski P, Crowther AJ, Jiménez LA, et al. The effect of smoking on the transcriptional regulation of lung inflammation in patients with chronic obstructive pulmonary disease. Am J Respir Crit Care Med 2006;174:41-50.

14. MacNee W. Oxidants/antioxidants and chronic obstructive pulmonary disease: pathogenesis to therapy. Novartis Found Symp 2001;234:169-85; discussion 185-8.

15. Wagner PD. Possible mechanisms underlying the development of cachexia in COPD. Eur Respir J 2008;31:492-501. 